\title{
Sequence and intramolecular distance scoring analyses of
}

\section{microbial rhodopsins [version 1; peer review: 2 approved]}

\author{
Miki Asano, Shunta Ide, Atsushi Kamata, Kiyohiro Takahasi, Tetsuji Okada
}

Department of Life Science, Gakushuin University, Tokyo, Japan

\author{
V1 First published: 12 Feb 2016, 5:165 \\ https://doi.org/10.12688/f1000research.7920.1 \\ Latest published: $06 \mathrm{Apr}$ 2016, 5:165 \\ https://doi.org/10.12688/f1000research.7920.2
}

\section{Abstract}

Recent accumulation of sequence and structural data, in conjunction with systematical classification into a set of families, has significantly advanced our understanding of diverse and specific protein functions. Analysis and interpretation of protein family data requires comprehensive sequence and structural alignments. Here, we present a simple scheme for analyzing a set of experimental structures of a given protein or family of proteins, using microbial rhodopsins as an example. For a data set comprised of around a dozen highly similar structures to each other (overall pairwise root-mean-squared deviation < 2.3 $\AA$ ), intramolecular distance scoring analysis yielded valuable information with respect to structural properties, such as differences in the relative variability of transmembrane helices. Furthermore, a comparison with recent results for $\mathrm{G}$ protein-coupled receptors demonstrates how the results of the present analysis can be interpreted and effectively utilized for structural characterization of diverse protein families in general.

Keywords

Membrane, receptor , opsin , crystallography , coordinates

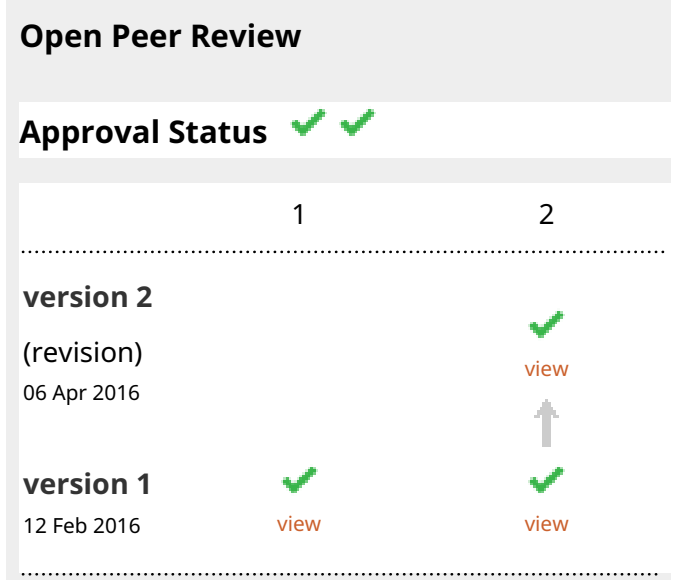

1. Javier V. Navarro, University of Texas

Medical Branch, Galveston, USA

2. Minoru Sugihara, Meiji Pharmaceutical

University, Tokyo, Japan

Any reports and responses or comments on the article can be found at the end of the article.

Corresponding author: Tetsuji Okada (tetsuji.okada@gakushuin.ac.jp)

Competing interests: No competing interests were disclosed.

Grant information: Supported by the Ministry of Education, Culture, Sports, Science and Technology of Japan (S1312002 to Department of Life Science, Gakushuin University).

The funders had no role in study design, data collection and analysis, decision to publish, or preparation of the manuscript.

Copyright: @ 2016 Asano $\mathrm{M}$ et al. This is an open access article distributed under the terms of the Creative Commons Attribution License , which permits unrestricted use, distribution, and reproduction in any medium, provided the original work is properly cited. Data associated with the article are available under the terms of the Creative Commons Zero "No rights reserved" data waiver (CCO 1.0 Public domain dedication).

How to cite this article: Asano M, Ide S, Kamata A et al. Sequence and intramolecular distance scoring analyses of microbial rhodopsins [version 1; peer review: 2 approved] F1000Research 2016, 5:165 https://doi.org/10.12688/f1000research.7920.1

First published: 12 Feb 2016, 5:165 https://doi.org/10.12688/f1000research.7920.1 


\section{Introduction}

Microbial rhodopsins (MRs) are retinal proteins found in archaea, bacteria and eukaryotic algae. They share a common architecture including a heptahelical transmembrane (7TM) bundle and function as either light-dependent proton/ion transporters or photon sensors. Recent introduction of these proteins to brain research has substantially advanced our understanding of neuronal functions ${ }^{1,2}$. As a prototypical member of this family, bacteriorhodopsin (bR) and its proton-pumping mechanism have been studied extensively over the past forty years ${ }^{3,4}$. There are more than 130 wild type and mutant crystal structure entries of bR deposited in the Protein Data Bank (PDB). In addition to other retinal proteins found early in archaea, such as halorhodopsins and sensory rhodopsins, recent studies have demonstrated the presence of a number of proteins belonging to MR family in a wide range of organisms ${ }^{5,6}$. Crystal structures obtained for some of these proteins have shown that the arrangement of the seven helices is conserved ${ }^{7,8}$, and their 7TM domains are valuable for examinations of the effects of experimental conditions and sequence variation on structure.

Another class of well-known 7TM proteins are G protein-coupled receptors (GPCRs), for which more than 120 crystal structure entries are available in PDB. They all activate heterotrimeric G proteins upon agonist binding, but their seven helices exhibit significant divergence, reflecting a high degree of ligand variation, from small amines to peptide hormones ${ }^{9,10}$. A recent study demonstrated that, despite such variation among GPCRs, some conserved features in terms of intramolecular atomic distances were discernible ${ }^{11}$. This observation was based on a systematic analysis of $\mathrm{C}_{\alpha}-\mathrm{C}_{\alpha}$ distances in crystal structures archived in PDB and hereafter we refer to this method as distance scoring analysis (DSA). For DSA, scoring of distance conservation among a set of crystal structures is simply made by taking the inverse of the coefficient of variation, wherein this coefficient is the average divided by the standard deviation.

If the number of available structures for this analysis was enough, it would be expected that structural differences due to either experimental conditions or sequence variation could be separately evaluated. In the previous analysis on GPCRs, we mainly focused on how the scores for $\mathrm{C}_{\alpha}-\mathrm{C}_{\alpha}$ distances in the 7TM bundle change as more variation in sequence was included because the apparent structural differences among receptors from different classes (rhodopsin-like, and others) were so large $\mathrm{e}^{11}$.

In the present study, we show that the DSA approach previously applied to GPCRs is also useful for highlighting bR and other MR helical regions that are relatively insensitive to the factors possibly affecting 7TM bundle structures. From the analysis of wild-type dark-state bR structures, we found that crystal packing could affect variability of a specific region of the 7TM bundle. On the other hand, the analysis of all MRs of known structure suggests that the regions involving high-score $\mathrm{C}_{\alpha}-\mathrm{C}_{\alpha}$ distances appear to be highly correlated with the functional importance. Furthermore, a comparison between two classes of 7TM proteins, MRs and GPCRs, demonstrates how the present analysis can be applied to diverse proteins families in general.

\section{Results}

Dataset 1. Raw data for DSA (Figure 2-Figure 6, Figure S3-Figure S6)

http://dx.doi.org/10.5256/f1000research.7920.d113285

Dataset 2. Python script for making a score vs distance plot

http://dx.doi.org/10.5256/f1000research.7920.d113889

\section{Sequence analysis}

Aside from the conventional serial numbering of polypeptide amino acids from the amino terminus, a common numbering system for a set of proteins based on conserved positions is expected to facilitate comparative protein family studies. A remarkable example involves GPCRs, for which an amino acid position in 7TM helices is given a common number (a BW number) ${ }^{12}$. For example, the most highly conserved asparagine in helix I is referred to as 1.50 and the other residues in the helix are numbered in descending order toward the amino-terminal side or increasing order toward the carboxylterminal side. Thus, our selection of helix I in the previous analysis corresponds to a polypeptide range of 1.35 to 1.59 (25 residues). Such a clear definition of polypeptide positions is very important for the quantitative analysis of structures that have different underlying sequences.

Since no such numbering scheme has been proposed for MRs, we first analyzed the amino acid sequences for this family archived in the InterPro database (www.ebi.ac.uk/interpro/) and identified the most conserved position in each of 7TM helices. Based on 603 sequences that include archaeal (178), bacterial (182), and algal (243) retinal proteins, an alignment was created and the distribution of amino acid types at each position was obtained, as shown in Table S1. The most highly conserved residue position in each helix was identified (Figure S1) and assigned a number, *.50, in which “*” indicates a letter for helix identification. Since helix assignment with $A$ to $G$ has been frequently used for MRs, we follow this convention for helix description of this family. However, for residue numbering, we use numerals 1 to 7 for "** in the present study in order to avoid confusion with single letter representation of amino acids.

Since we only considered possible retinal proteins, the amino acid type at 7.50 was Lys, and it exhibited $100 \%$ conservation (Table 1). Helix F contained three highly conserved residues, which we designated 6.50 (Trp), 6.53 (Tyr), and 6.54 (Pro). The degree of conservation was very similar for 6.50 and 6.53 , and higher than $97 \%$ among the 603 sequences. Helix $\mathrm{C}$ also contained a set of positions that exhibited greater than $95 \%$ conservation. At all these positions, the amino acid types, except 5.50 in helix E, were identical among the 13 MRs examined by DSA in the present study (Figure S2).

\section{Data selection}

As of February 5_2015, there were 135 entries for MRs in PDB and the contents are summarized on our website (www.gses.jp), which 
Table 1. The 7TM bundle of 170 residues used for the present study and the proposed common numbering for microbial retinal proteins.

\begin{tabular}{|c|c|c|c|c|}
\hline Helix & $\begin{array}{c}\text { Common } \\
\text { numbering }\end{array}$ & $*$.50 in bR & $\begin{array}{c}\text { Numbering } \\
\text { in bR }\end{array}$ & $\begin{array}{c}\text { Helix } \\
\text { length }\end{array}$ \\
\hline A & $1.34-1.55$ & Phe27 & $11-32$ & 22 \\
\hline B & $2.33-2.56$ & Tyr57 & $40-63$ & 24 \\
\hline C & $3.46-3.67$ & Arg82 & $78-99$ & 22 \\
\hline D & $4.33-4.56$ & Gly122 & $105-128$ & 24 \\
\hline E & $5.30-5.57$ & Leu152 & $132-159$ & 28 \\
\hline F & $6.36-6.60$ & Trp182 & $168-192$ & 25 \\
\hline G & $7.36-7.60$ & Lys216 & $202-226$ & 25 \\
\hline
\end{tabular}

does not include redundant or outdated structures. By examining the superimposed chains from various proteins, we selected a range of amino acids for each of the seven helices with at least 22 residues per helix ( 6 turns for regular geometry), resulting in a total of 170 residue bundles. Thus, we considered $14,365 \mathrm{C}_{\alpha}-\mathrm{C}_{\alpha}$ pairs per 7TM bundle for the present analysis. From this archive, we made several data sets containing different combinations of polypeptide chains. Set 1 consisted of 9 chains of wild-type, dark-state bR, each of which represents a structure solved in a distinct space group or by a different research group. Set 2 (Figure 1A) was more redundant than set 1 , including multiple chains per entry, resulting in a total of 22 chains (Table S2). Set 3 contained a set of 13 chains (Figure 1B), each from structures with a unique sequence, as shown in Table 2. The other sets included, for instance, bR mutants, dark-state halorhodopsins and sensory rhodopsins. The results for these sets, other than 1 to 3, will be described elsewhere (Ono et al. unpublished report).

\section{Distance analysis for bR structures}

In all PDB entries for MRs, the most abundant structure was bacteriorhodopsin from Halobacterium salinarum. Thus, we are interested in determining how effective DSA is in detecting the intramolecular structural conservation among the ground-state wild-type bRs. The superimposed projection view of 22 chains in set 2 is shown in Figure 1A. These are obviously very similar to each other and are within the overall pairwise root-mean-squared deviation of $1.2 \AA$ for $170 \mathrm{C}_{\alpha}$ positions (Table S4). This similarity corresponds to a pairwise correlation coefficient of more than 0.993 calculated for the $14,365 \mathrm{C}_{\alpha}-\mathrm{C}_{\alpha}$ distances.

DSA results obtained from these 22 chains in set 2 and from 9 chains in set 1 are shown in Figures 2B and 2A, respectively. Scores for $\mathrm{C}_{\alpha}-\mathrm{C}_{\alpha}$ distances estimated by DSA are defined as the inverse of the coefficient of variation ${ }^{11}$, and should be higher when the variation among chains is smaller. The plot that includes all 14,365 points demonstrates the distribution of scores against the average distances. The overall pattern depicted in these plots is in contrast to a previous report for GPCRs ${ }^{11}$ and the updated analysis (Figure S3). In the case of GPCRs of various sequences, populations with high scores are dominated by the contribution from intrahelical
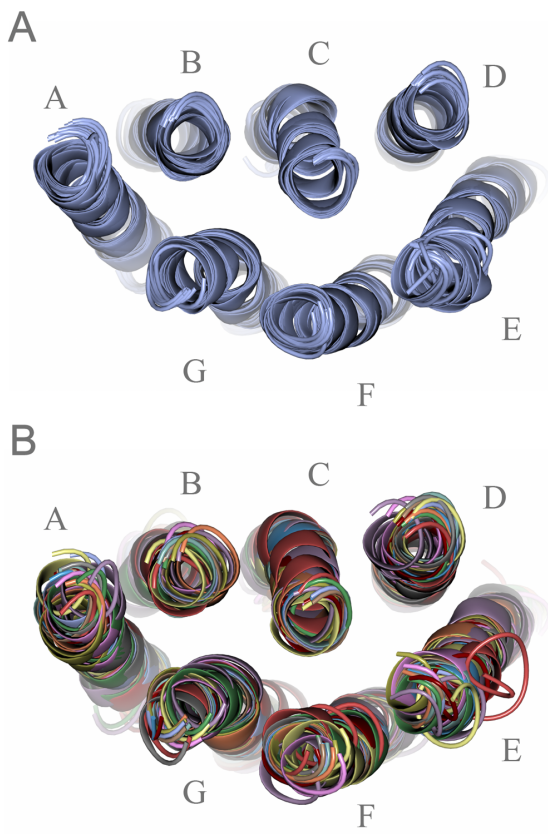

Figure 1. Graphical representation of MRs with known structure. A. 22 polypeptide chains in set 2 (dark-state wild-type bR structures) and B. 13 unique chains in set 3 (MRs of different sequences).

Table 2. The crystallographic models used for set 3 in the present study.

\begin{tabular}{|l|l|l|l|}
\hline PDB ID & Protein & Species & Domain \\
\hline 1PY6 & Bacteriorhodopsin & H. salinarum & archaea \\
\hline 1E12 & Halorhodopsin & H. salinarum & archaea \\
\hline 3A7K & Halorhodopsin & N. pharaonis & archaea \\
\hline 1H68 & Sensory Rhodopsin II & N. pharaonis & archaea \\
\hline 1XIO & $\begin{array}{l}\text { Anabaena Sensory } \\
\text { Rhodopsin }\end{array}$ & $\begin{array}{l}\text { Nostoc sp. PCC } \\
\text { 7120 }\end{array}$ & bacteria \\
\hline 1UAZ & Archaerhodopsin-1 & H. chaoviator & archaea \\
\hline 2EI4 & Archaerhodopsin-2 & H. chaoviator & archaea \\
\hline 3DDL & Xanthorhodopsin & S. ruber & bacteria \\
\hline 3AM6 & $\begin{array}{l}\text { Acetabularia } \\
\text { Rhodopsin II }\end{array}$ & A. acetabulum & eukaryota \\
\hline 4HYJ & $\begin{array}{l}\text { Proteorhodopsin } \\
\text { (green) }\end{array}$ & E. sibiricum & bacteria \\
\hline 4KLY & $\begin{array}{l}\text { Proteorhodopsin } \\
\text { (blue) }\end{array}$ & HOT75 & bacteria \\
\hline 4FBZ & $\begin{array}{l}\text { Deltarhodopsin-3 } \\
\text { 4 H. thermotolerans }\end{array}$ & archaea \\
\hline 4L35 & Cruxrhodopsin-3 & H. vallismortis & archaea \\
\hline
\end{tabular}

pairs, whereas interhelical pairs exhibit high scores in the bR sets. This result for bR shows that interhelical residue pairs exhibit high scores in a set containing very similar chains, and also suggests that external factors such as crystal lattice packing and solvent conditions that possibly affect the structures tend to highlight 

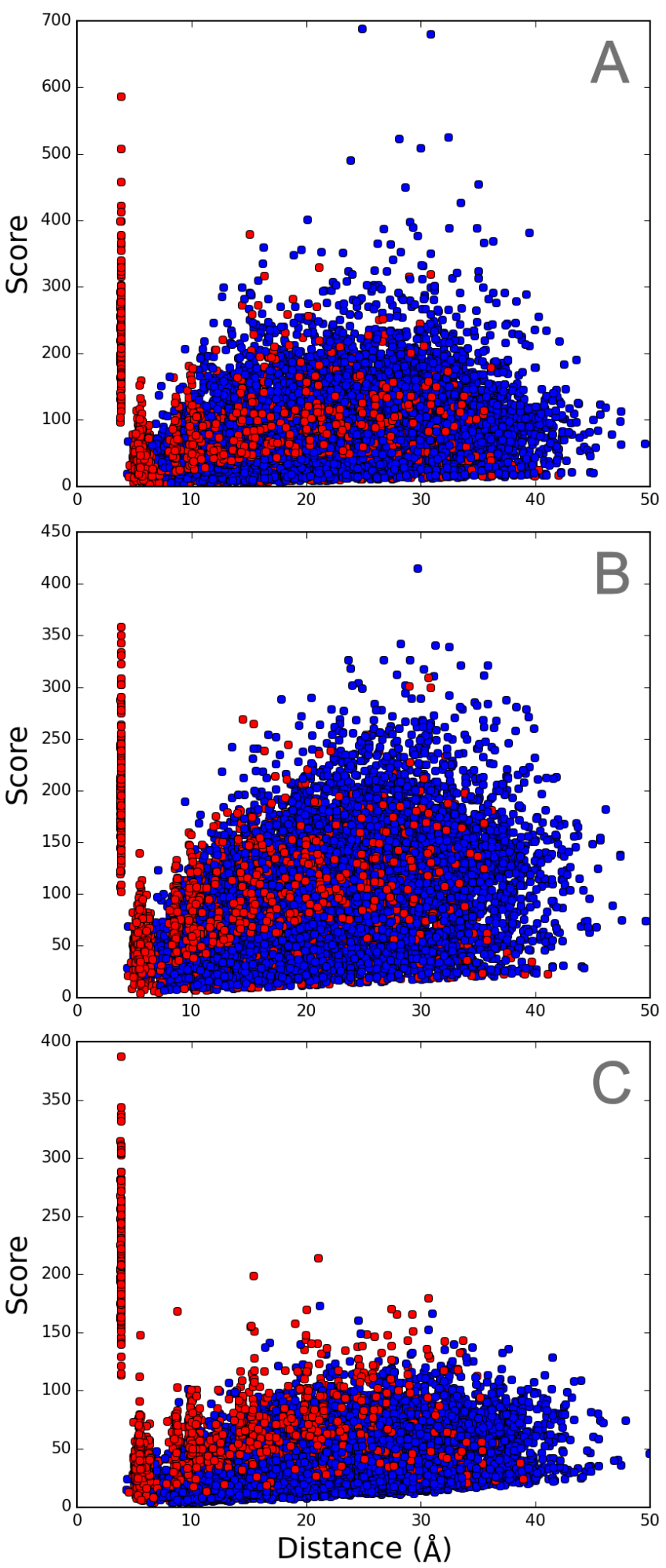

Figure 2. Correlation between score and the average distance for the 14,365 C - C pairs. A. set $1, B$. set 2 , and C. set 3 . Intrahelical and interhelical $\mathrm{C}_{\alpha}-\mathrm{C}_{\alpha}$ pairs are colored in red and blue, respectively. single helix geometry changes rather than changes in interhelical arrangements. A comparison between the results for set 1 and 2 indicates that high scores are biased toward longer distances for the interhelical pairs in set 2 . This may result from the inclusion of highly similar structures (Table S4) in set 2.

When the intrahelical components were examined in detail, some pairs with high scores were found to originate from helices B and $\mathrm{D}$ in both sets 1 (Figure 3) and 2 (Figure S4). This finding is more clearly demonstrated by the cumulative numbers (expressed as ratios relative to the total number) of the $\mathrm{C}_{\alpha}$ pairs ranked in the top 1,000 (Figure 3, lower panels). This feature of helices $\mathrm{B}$ and $\mathrm{D}$ is in contrast to the nearby helices $\mathrm{A}$ and $\mathrm{C}$, for which few pairs appear in the top 1,000 ranks. Pairs with the highest scores for helix $\mathrm{B}$ were between the residues of inward-facing intracellular region and the residues of lipid-facing extracellular region, and for helix D involved the cytoplasmic (amino) terminal residues. The implications of these findings will be discussed later.
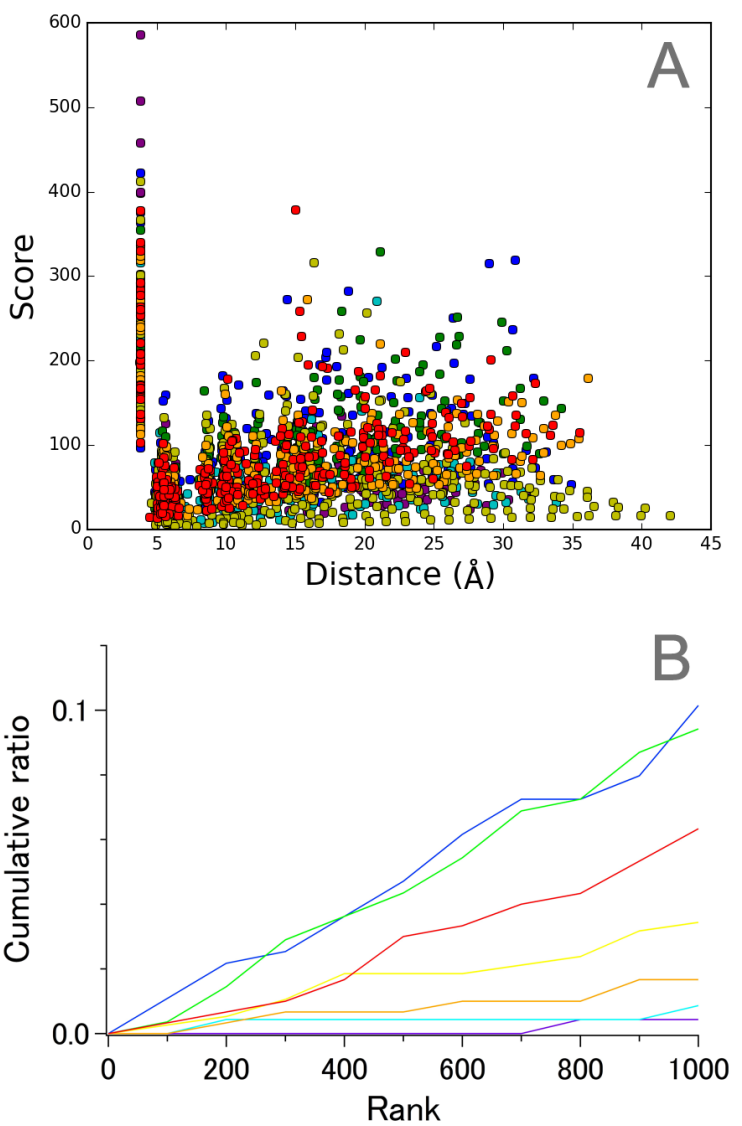

Figure 3. DSA results for 1,992 intrahelical pairs in set 1 . A. Correlation between score and average distance. B. Cumulative ratio of the number of $\mathrm{C}_{\alpha}-\mathrm{C}_{\alpha}$ pairs in the top-ranked 1,000. The pairs are colored as follows; purple, helix A; blue, helix B; cyan, helix C; green, helix D; yellow, helix E; orange, helix F; red, helix G. 
To examine whether useful information can be obtained by analyzing interhelical components, we first checked the distance dependence of scores. In principle, this is easily done when a comparison is made among the helix pairs such as A-B, A-C, and A-D, the latter of which contains longer-distance pairs. As shown in Figure S5A, it is apparent that A-D pairs tended to exhibit higher scores than $\mathrm{A}-\mathrm{B}$, and $\mathrm{A}-\mathrm{C}$ in the case of set 2 . Therefore, a baseline correction or comparison of scores within a limited range of distances should be made when evaluating the pairs with high scores in such cases. When we compare helix pairs of similar distances, like A-B, B-C, and $\mathrm{C}-\mathrm{D}$, however, such distance dependence was not obvious (Figure S5B) and some remarkably high scores are found for B-C pairs. Importantly, more conserved B-C pairs were discernible even when the number of chains considered was limited to 9 as in set 1 (Figure S5C), which contains chains of either different space groups or research groups who solved the structure (Table S2). The high score B-C pairs were between the residues of lipid-facing extracellular region in helix $\mathrm{B}$ and the residues of intracellular region in helix $\mathrm{C}$. The former is consistent with the above-mentioned results for intrahelical pairs and the latter contains a cluster of leucines and Asp96 (3.64) which is implicated to be important for proton pumping function. From these results, we suggest that just under 10 chains of very similar structures can provide statistically significant information regarding the relatively insensitive intramolecular spacing of a protein against external forces.

\section{Distance analysis of MR structures}

The results for wild-type bR ground-state chains demonstrate how DSA scores represent intramolecular distance changes against environmental factors even in the absence of sequence variation. On the other hand, analysis of set 3 , which contains 13 chains of unique MR sequences, is expected to clarify the part of 7TM that is the most structurally conserved among the evolutionally related proteins. Although the number of available chains is fewer than the previously examined sets of GPCRs, we found that the overall pattern observed for all 14,365 pairs (Figure 2C) was more similar to that of 18,915 pairs of GPCRs (Figure S3A) than that of 14,365 pairs of dark-state wild-type bR (Figure 2A, 2B). This observation confirms that the contribution of interhelical pairs to the high-score population becomes insignificant when sequence variation is involved.

The most prominent intrahelical pairs with high scores were from helix G (Figure 4), to which retinal chromophore is attached. This finding is reasonable if we consider that all 13 proteins require retinal binding to a specific site, $\operatorname{Lys}(7.50)$, for their function as photoreceptors. Interestingly, the middle of this helix contains a $\pi$ bulge within which Lys(7.50) resides (Table S5). Thus, it appears that intrahelical distance conservation is not dependent on whether a helix assumes a regular geometry or not. This finding adds an important revision to the previous view that the remarkably high score observed for helix III in the 7TM bundle of GPCRs might be partly explained by its regular helical structure ${ }^{11}$.

It should also be noted that helix $\mathrm{C}$ appeared to be the most variable among the seven helices of MRs (Figure 4B). This was rather unexpected taking into account the fact that this helix contains highly conserved residues in addition to $\operatorname{Arg}(3.50)$, including $\operatorname{Tyr}(3.51), \operatorname{Trp}(3.54)$, and Pro(3.59) (Table S1), and these residue
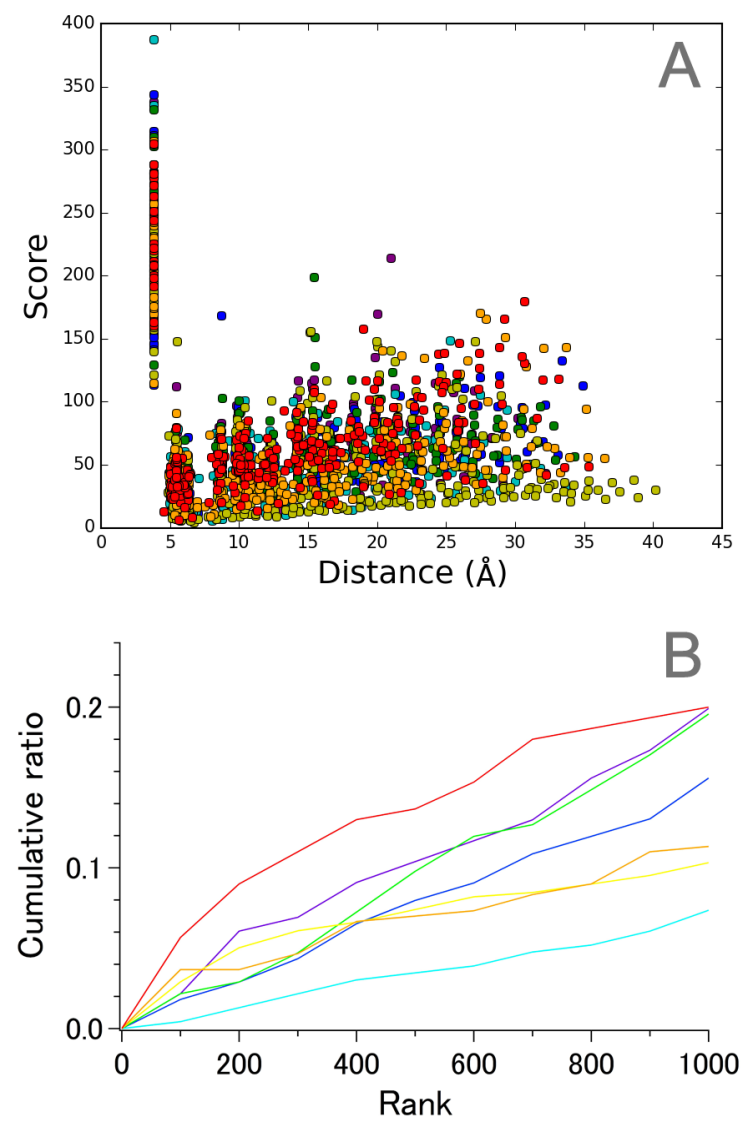

Figure 4. DSA results for 1,992 intrahelical pairs in set 3 . A. Correlation between score and average distance. B. Cumulative ratio of the number of $\mathrm{C}_{\alpha}-\mathrm{C}_{\alpha}$ pairs in the top-ranked 1,000. Coloring of the plots is the same as that in Figure 3.

types are completely conserved in 13 chains examined here by DSA (Figure S2). These observations suggest that intramolecular distance conservation among a set of evolutionally related proteins cannot always be inferred from the degree of sequence conservation. The structurally variable nature of helix C among 13 MRs may be in line with the finding that it does not contain many high score pairs in top 1000 ranks of dark-state wild-type bR sets (Figure 3). Another possible explanation for low scores of the pairs in helix $\mathrm{C}$ appears to be a substantial displacement in the backbone position in 2 halorhodopsin chains around the 3.53 position (Asp in most MRs, and Thr in $2 \mathrm{hRs}$ ), whereas an Asp to Asn mutation at this position in the structure of blue-absorbing proteorhodopsin (D97N) does not affect the structure of this region significantly.

As Figure 2C demonstrates, there was little distance dependency among the interhelical pairs in set 3; therefore, we examined the pairs in detail and noticed that a remarkable contribution to the high scores was attributed to the pairs between helices C and G (Figure 5, cyan). Since other interhelical pairs did not exhibit significant features, only E-G pairs are colored in yellow as a reference. The pairs with the highest scores involved the residues on the intracellular side 
of helix C and extracellular side of helix G, as shown in Figure 6. Relatively conserved spacing between these two regions is likely to ensure the binding and Schiff base protonation of all-trans-retinal chromophores to the cavity within a 7TM bundle of all MRs of known structure.

\section{Discussion}

\section{External factors affecting crystal structure}

In the present study, we first examined how different crystallization conditions affect the structure of ground-state wild-type bR. We used 22 chains for this purpose, the resolutions of which ranged from 1.8 to 3.5 , including 2 chains obtained by cryo-electron microscopy. These structures were solved in different solvent environments and lattice packing. Obvious differences among 22 chains were discernible mainly at the cytoplasmic terminal region of helix $\mathrm{E}$ by visual inspection after superimposition (Figure 1A). This observation appears to explain why pairs with very low scores come mostly from this particular helix (Figure 3A, yellow). On the other hand, other regions in the 7TM bundle exhibit only moderate deviation, so our quantitative study by DSA is expected to work well for extracting information regarding structural conservation rather than variation.
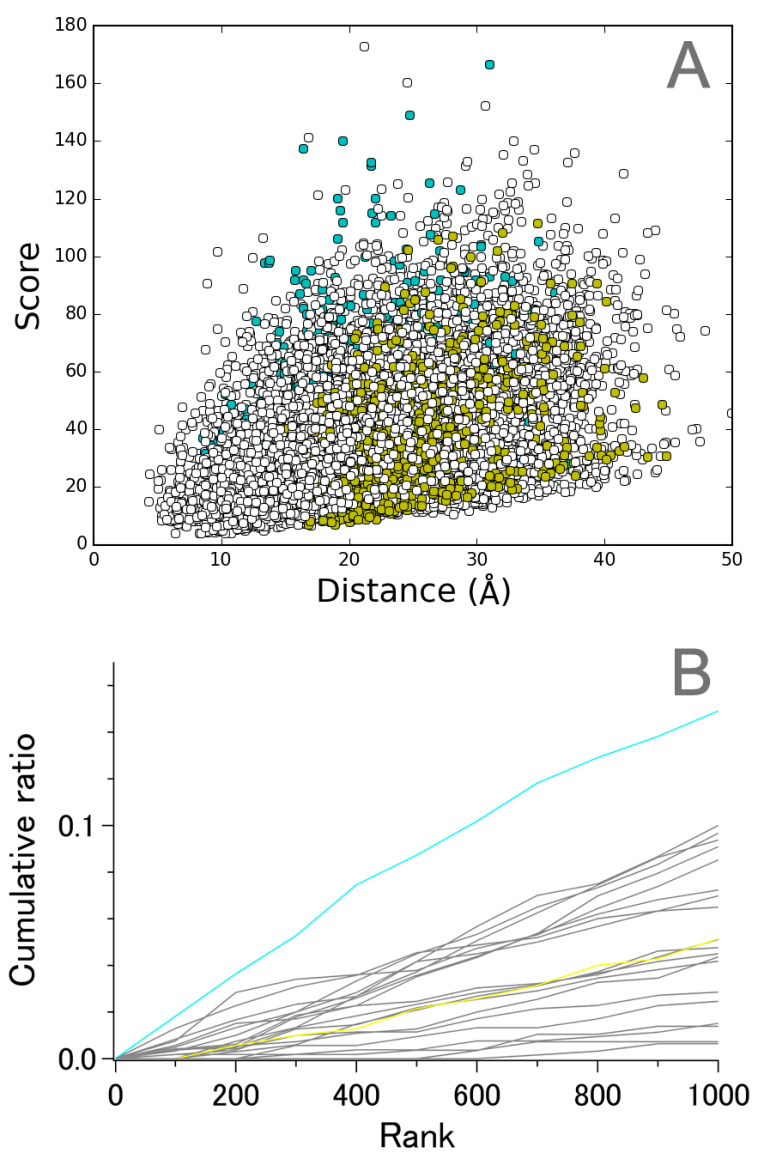

Figure 5. DSA results on 12,373 interhelical pairs in set 3 . A. Correlation between score and the average distance. B. Cumulative ratio of the number of $C_{\alpha}-C_{\alpha}$ pairs in the top-ranked 1,000. The pairs are colored as follows; cyan, C-G; yellow, E-G; gray, others.
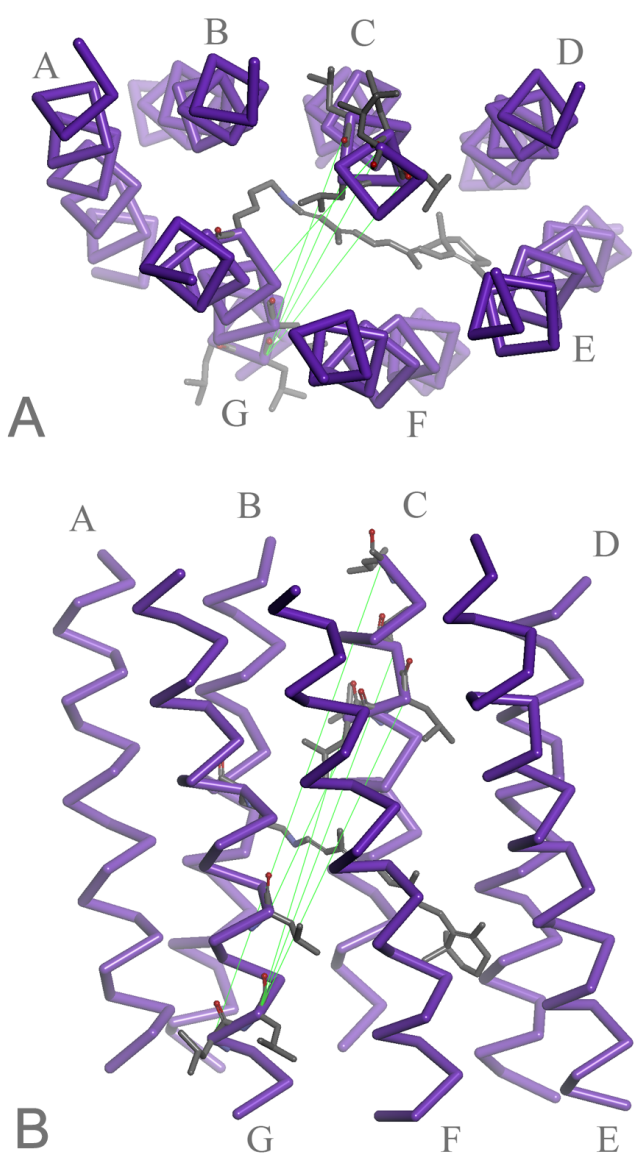

Figure 6. Graphical representation of conserved interhelical distances in set 3 including 13 unique bundles from all MRs with known structure. A. Top view from the cytoplasmic side. B. Side view from helices $F$ and $G$. The pairs between helices $C$ and $G$ with high scores are connected by green lines drawn on chain $A$ of 1PY6 (bR). A retinal chromophore attached to Lys(7.50) is also shown in the center.

Our finding that helix B is the most insensitive to external factors may reflect its inherent properties. A previous simulation study on the individual helices of bR suggested that the structures of helices $\mathrm{A}, \mathrm{B}$, and $\mathrm{E}$ are stable in sodium dodecyl sulfate micelles ${ }^{13}$. Another possibility is that helix B does not suffer from crystal packing effect. To address this, we examined the molecular arrangement in all 6 space groups. In 5 of the 6 space groups, including native P3 observed by electron microscopy on purple membranes, lateral interactions between helices B and D were found. Therefore, pairs with high scores found in these two helices (Figure 3) may reflect a stabilization effect owing to crystal lattice contact. Alternatively, inherently stable parts of helices B and D might contribute to the preference of trimeric arrangement for bR by providing suitable intermolecular interactions.

Considering that helix $\mathrm{C}$ contains a few residues that are important for the proton-pumping function of $\mathrm{bR}^{14,15}$, such as Asp85 (3.53) and Asp96 (3.64), it may sound curious that this helix does not contribute to pairs with high scores in sets 1 and 2. In fact, removal 
of a chain that exhibits distinct features can substantially affect the results and result in higher scores for some pairs in helix $\mathrm{C}$ (Figure S6) in set 1 ( 9 chains) but not in set 2 ( 22 chains). Therefore, careful examination of each data set is required especially when the number of chains is limited.

\section{Conservation among MRs}

We further performed DSA on the crystallographic models of $13 \mathrm{MRs}$, the sequences of which vary. The pairwise sequence identity (Table S3) ranges from $18.2 \%$ (between anabaena sensory rhodopsin and blue-absorbing proteorhodopsin) to $88.8 \%$ (between archaerhodopsin-1 and 2). This variation was less than that observed among previously analyzed and updated GPCRs (Table S3). Accordingly, the overall pairwise root-mean-squared deviation was smaller among the 13 MRs ( 2.3 $\AA$ at most between xanthorhodopsin and blue-absorbing proteorhodopsin) than among GPCRs ( $6 \AA$ at most between PAR1 thrombin receptor and CRF1 receptor) (Table S4) and this is reflected in the relatively higher scores in MRs than GPCRs (Figure 2C, Figure S3). However, both sets exhibited higher scores for intrahelical residue pairs than interhelical pairs, the latter of which might be more affected by sequence variation.

The high distance conservation between pairs in helices $\mathrm{C}$ and $\mathrm{G}$ found in the present study suggests that the DSA procedure is useful for detecting structural conditions necessary for common functionality of evolutionally related proteins. Whereas it appears that a slight distance dependency of scores may exist (Figure 5A), the largest contribution to populations exhibiting high scores for the pairs between helices $\mathrm{C}$ and $\mathrm{G}$ is not likely explained by such an effect.

In the case of MRs, all members are required to ensure binding of all-trans-retinal molecules in a cavity surrounded by 7 TM helices. Whereas helices $\mathrm{C}$ and $\mathrm{G}$ are in contrast to each other with regard to the degree of intrahelical structural conservation (Figure 4), our results suggest that a strict condition of spacing between the cytoplasmic terminal region of helix $\mathrm{C}$ and the extracellular side of helix $\mathrm{G}$ must be fulfilled in all MRs (Figure 5, Figure 6). Interestingly, retinal Schiff base bound to the side chain of Lys(7.50) resides just in the middle of this conserved spacing (Figure 6). We suspect that definite structural requirement for MRs, whatever the functions are (pumps, channels, or sensors), would be proper relative positioning of $\operatorname{Lys}(7.50)$ and a set of residues from helix $\mathrm{C}$ which contribute significantly to holding of the retinal polyene chain and protonation of the Schiff base.

\section{Implications for the structural biology}

Intramolecular distance information from existing crystal structures has long been utilized in the field of structural biology for such purposes as domain recognition ${ }^{16}$, construction of new models ${ }^{17}$, and detection of conformational changes ${ }^{18}$. Although the DSA method might require further improvements, it can be applied in the current form, to the detailed mining of information from larger sets of data than previously examined, and specifically to a number of protein families given that reliable alignments can be obtained. Among the membrane proteins in $\mathrm{PDB}$, the largest category with more than
180 entries is ion channels that transport potassium, sodium, and protons. These proteins function as multi-subunit complexes and exhibit no similarity with any of the 7TM proteins. The second and third-most represented membrane protein families in PDB, MRs and GPCRs studied by DSA, had an advantage in that their alignments were rather straightforward. The present study suggests that around a dozen experimental structures with related and aligned sequences or obtained under distinct conditions can be used to infer statistically significant features of a protein or protein family. From this perspective, a structural archive would be a far more valuable source of information to improve our understanding of biological macromolecules.

\section{Methods}

Sequence analysis

Microbial rhodopsin sequences were obtained from InterPro (www.ebi.ac.uk/interpro/) v.48 under the classes archaeal/bacterial/fungal rhodopsin (IPR001425) and archaeal/bacterial/fungal rhodopsin-like (IPR029730). Archaeal proteins did not differ significantly between the two classes, while bacterial and eukaryotic proteins were highly enriched in the IPR029730 class. As the excess bacterial proteins in the IPR029730 class were mostly proteorhodopsins, the sequence set was constructed from the IPR001425 archaeal and bacterial proteins (518 and 298 sequences, respectively) and IPR029730 eukaryotic proteins (651 sequences). A multiple sequence alignment was performed with ClustalW ${ }^{19}$ implemented in BioEdit 7.2.5 ${ }^{20}$ for each of the three domains. Based on manual inspection of the results, misaligned or extremely short or long sequences were removed from each domain set. The results for each domain were then merged and an additional alignment was carried out. The distribution of amino acid types at each position was obtained using the Positional Amino Acid Numerical Summary function implemented in BioEdit.

\section{Distance analysis}

Crystallographic models of MRs were obtained from PDB (www.rcsb.org/pdb/) and classified manually as listed in our web site (www.gses.jp/7tmsp/) into several groups such as wild-type and mutant bRs, halorhodopsins, and sensory rhodopsins. These PDB entries (accession numbers are as noted in Table S2 Table S4) were processed to make single polypeptide chains and further truncated to 7TM bundles of 170 residues manually by Discovery Studio Visualizer 3.1 (Accelrys Inc.), ensuring that the alignments for different receptors were correct. The overall pairwise root-mean-squared deviation and correlation coefficient were obtained by Discovery Studio Visualizer 3.1 (Accelrys Inc.) and pca-excel 1.0 (ss-nakano Inc.), respectively. DSA was performed on the $\mathrm{C}_{\alpha} \mathrm{s}$ of the MR bundles as well as 23 GPCRs with unique sequences (19 rhodopsin-like and 4 non rhodopsin-like receptors), following a recently described procedure $^{11}$. Briefly, the average, standard deviation, and the inverse of coefficient of variation (score) of each $\mathrm{C}_{\alpha}$ pair distance were calculated in each of the sets (Dataset 1). The 7TM bundle of the P2Y12 receptor (PDB ID: 4NTJ) aligned to rhodopsin-like receptors was assumed to lack a residue at the amino terminus of helix VI (6.29). Similarly, the 7TM bundles of the class C mGluR1 (PDB ID: 4OR2) and mGluR5 (PDB ID: 4OO9) receptors were assumed to 
lack two residues at the carboxyl termini of helix II (2.66 and 2.67) and VI (6.59 and 6.60). The resulting number of $\mathrm{C}_{\alpha}$ pairs was 18,915 .

Score vs distance plots were prepared with matplotlib (matplotlib.org/) by implementing in an original python script for DSA (Dataset 2), and other graphs were drawn using Igor Pro 6.37 (WaveMetrix Inc.). Protein graphics were prepared with either CCP4MG 2.8.121 or Discovery Studio Visualizer 3.1 (Accelrys Inc.).

\section{Data availability}

F1000Research: Dataset 1. Raw data for DSA (Figure 2-Figure 6, Figure S3-Figure S6), 10.5256/f1000research.7920.d11328522

F1000Research: Dataset 2. Python script for making a score vs distance plot, 10.5256/f1000research.7920.d11388923

\section{Author contributions}

M.A., S.I., A.K., and T.O. performed analysis, K.T., and T.O. validated data, T.O. designed research, and wrote the manuscript.

\section{Competing interests}

No competing interests were disclosed.

Grant information

Supported by the Ministry of Education, Culture, Sports, Science and Technology of Japan (S1312002 to Department of Life Science, Gakushuin University).

I confirm that the funders had no role in study design, data collection and analysis, decision to publish, or preparation of the manuscript.

\section{Supplementary material}

extracellular side
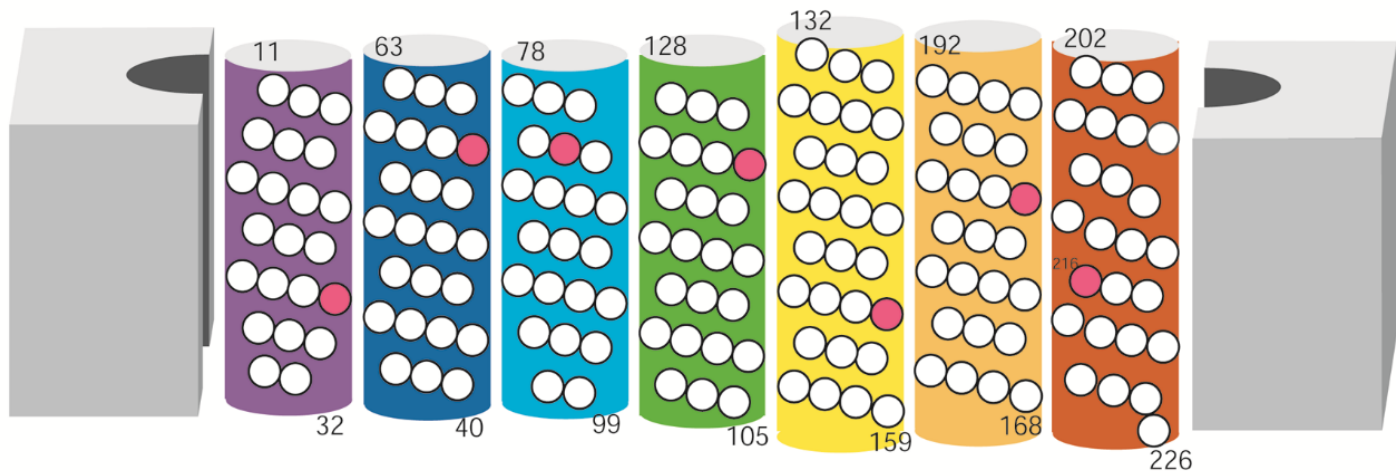

cytoplasmic side

Figure S1. Schematic drawing of the 7TM bundle of MRs showing the proposed position of the *.50 residue in each helix (colored pink). The numbers attached to the helices are the selected ranges in the bR sequence. 
A

B

$\mathrm{C}$

D

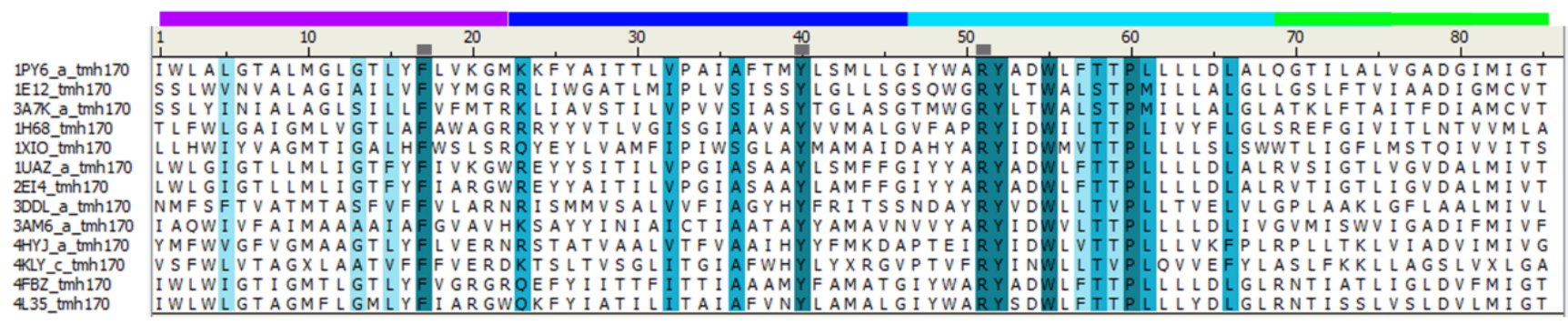

E

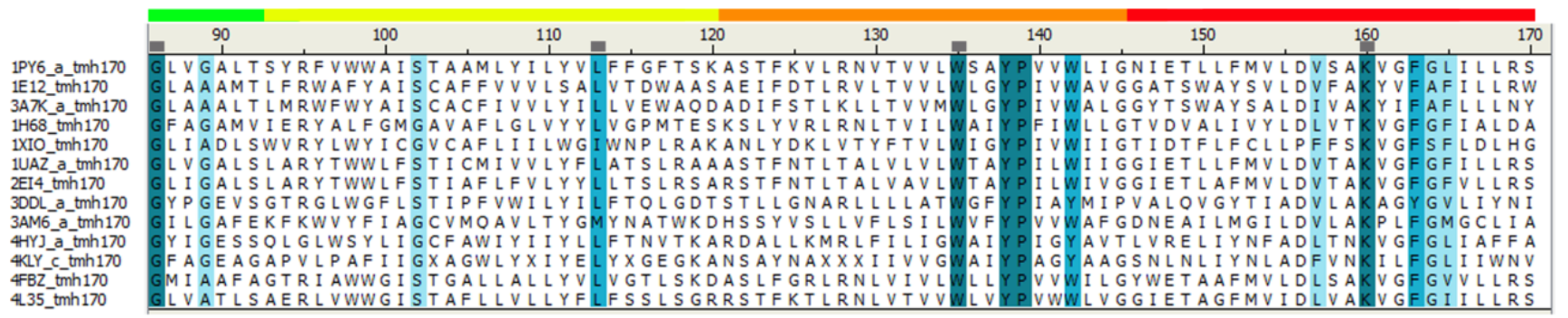

Figure S2. Sequence alignment of the 7TM regions of 13 MRs in set 3. The conserved positions are colored as follows: dark blue, identical; blue, strong similarity; light blue, weak similarity, according to PAM250 matrix definition. The small squares on the ruler indicate the *.50 positions of the numbering proposed for MRs. 
A

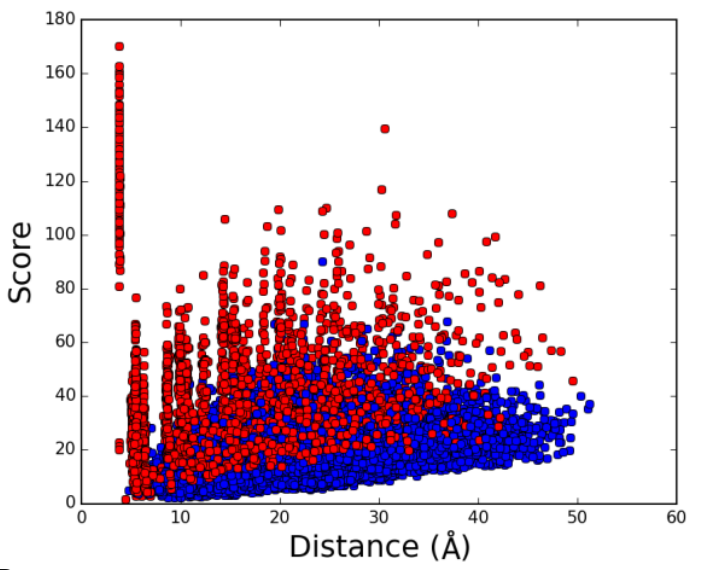

B
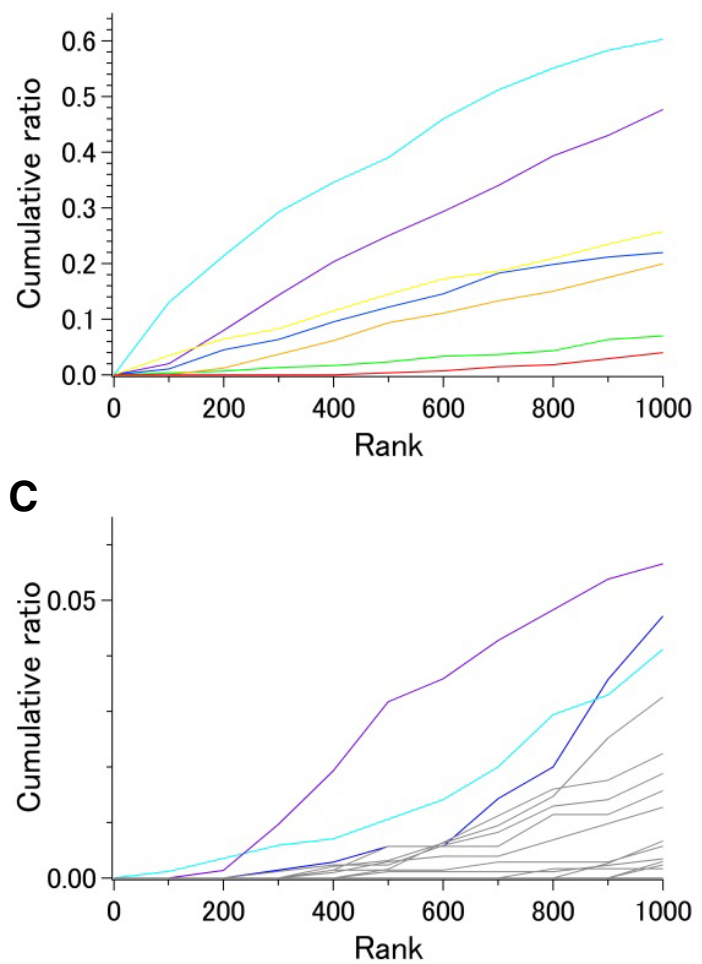

Figure S3. DSA results for all 18,915 $\mathrm{C}_{-}-\mathrm{C}_{a}$ pairs in the updated set of GPCRs containing 23 unique receptor chains. (A) Correlation between score and average distance. Intrahelical and interhelical $\mathrm{C}_{\alpha}-\mathrm{C}_{\alpha}$ pairs are colored in red and blue, respectively. (B) Cumulative ratio of the number of 2,661 intrahelical $C_{-}-C_{\alpha}$ pairs in the top-ranked 1,000. Coloring of the plots is the same as that used in Figure 3 . (C) Cumulative ratio of the number of 16,254 interhelical $C_{\alpha}-C_{\alpha}$ pairs in the top-ranked 1,000. Purple, I-VI; cyan, I-III, blue, I-II; gray, others. 
A

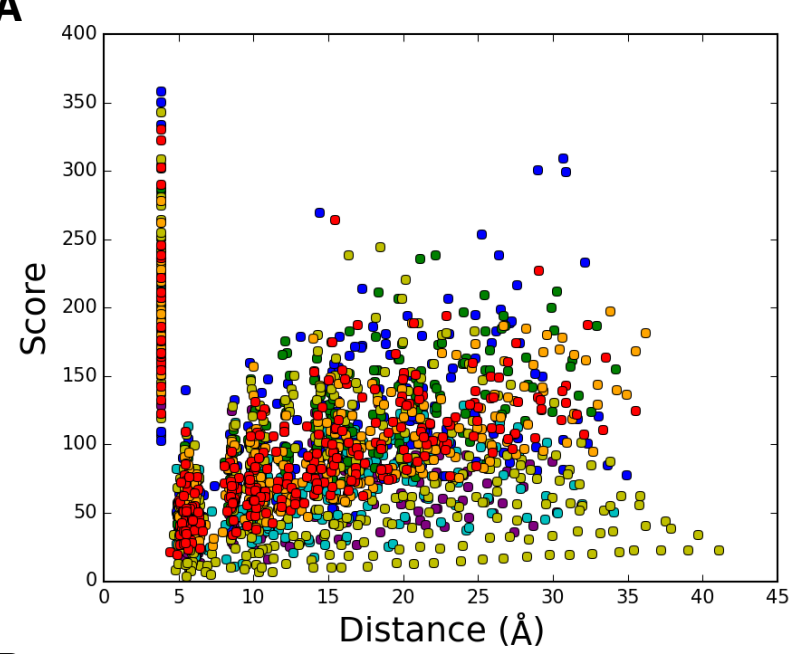

B

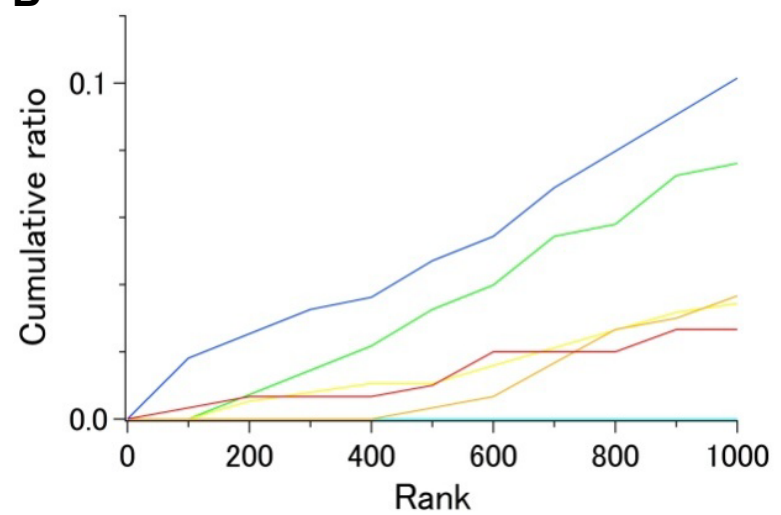

Figure S4. DSA results for 1,992 intrahelical pairs in set 2. (A) Correlation between score and average distance. (B) Cumulative ratio of the number of $\mathrm{C}-\mathrm{C}$ pairs in the top-ranked 1,000. The pairs are colored as follows; purple, helix $\mathrm{A}$; blue, helix $\mathrm{B}$; cyan, helix $\mathrm{C}$; green, helix D; yellow, helix E; orange, helix F; red, helix G. 
A

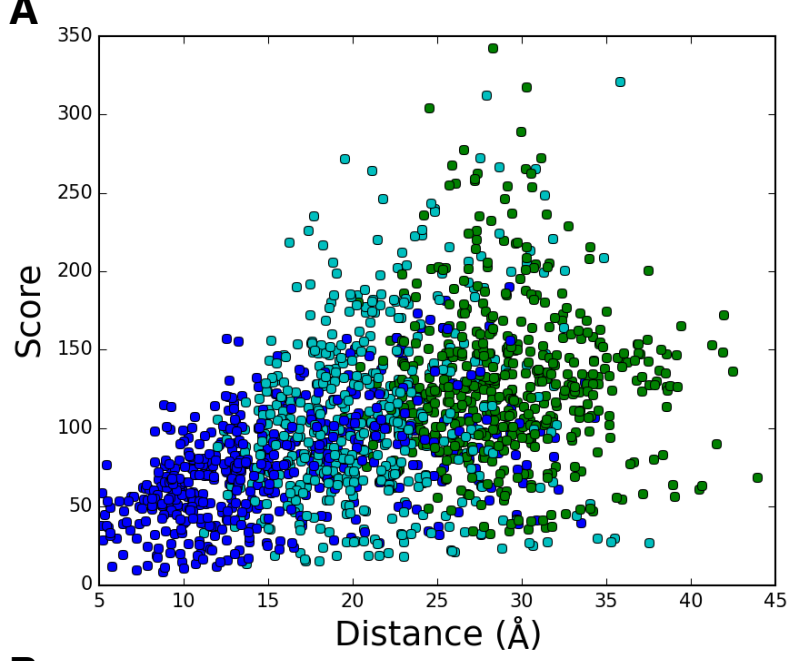

B

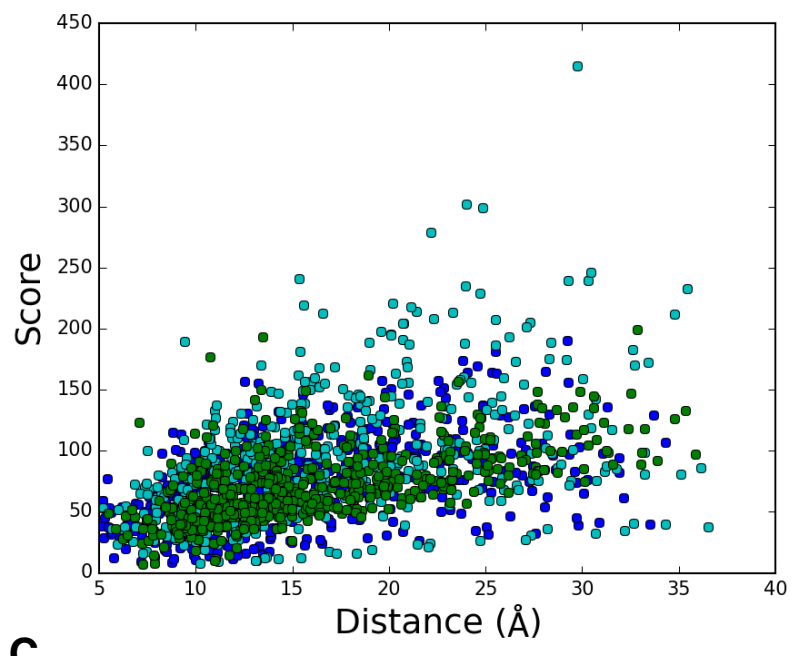

C

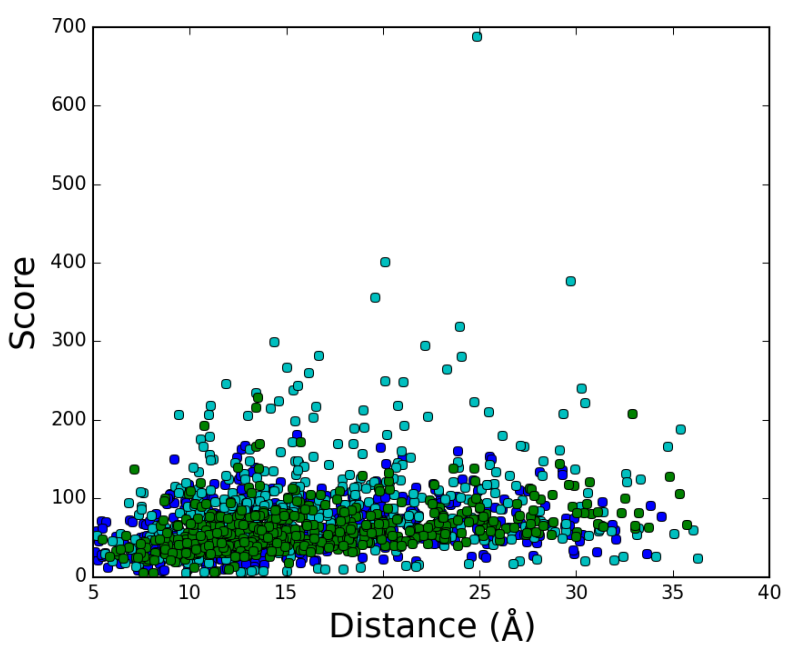

Figure S5. Correlation between score and average distance of a part of the interhelical pairs of bR structures. (A) set 2, blue, A-B; cyan, A-C; green, A-D. (B) set 2, blue, A-B; cyan, B-C; green, C-D. (C) set 1, blue, A-B; cyan, B-C; green, C-D. 
A

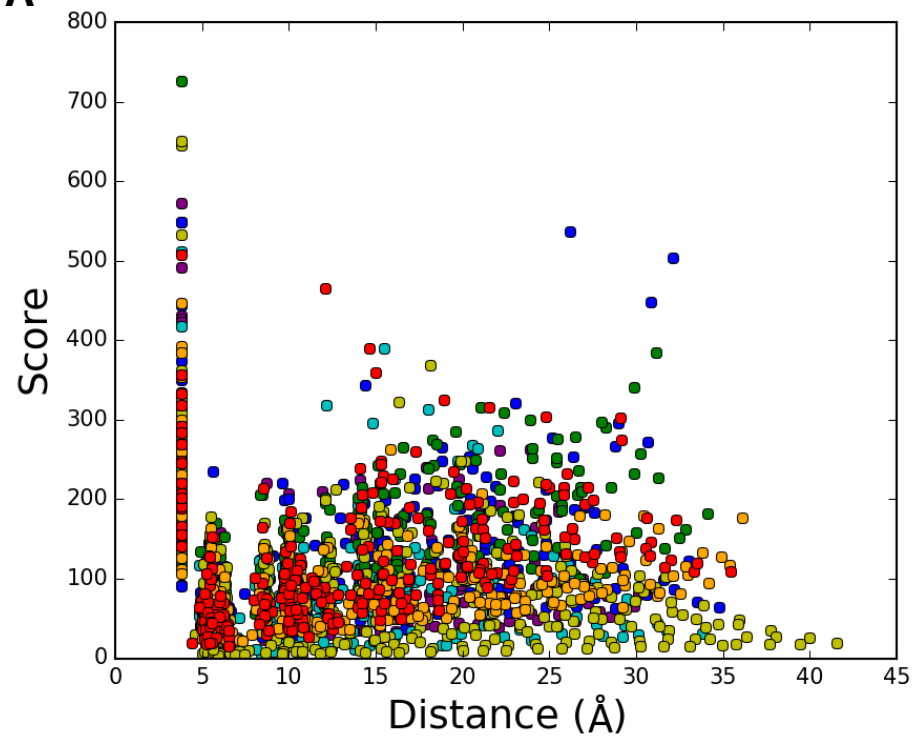

B

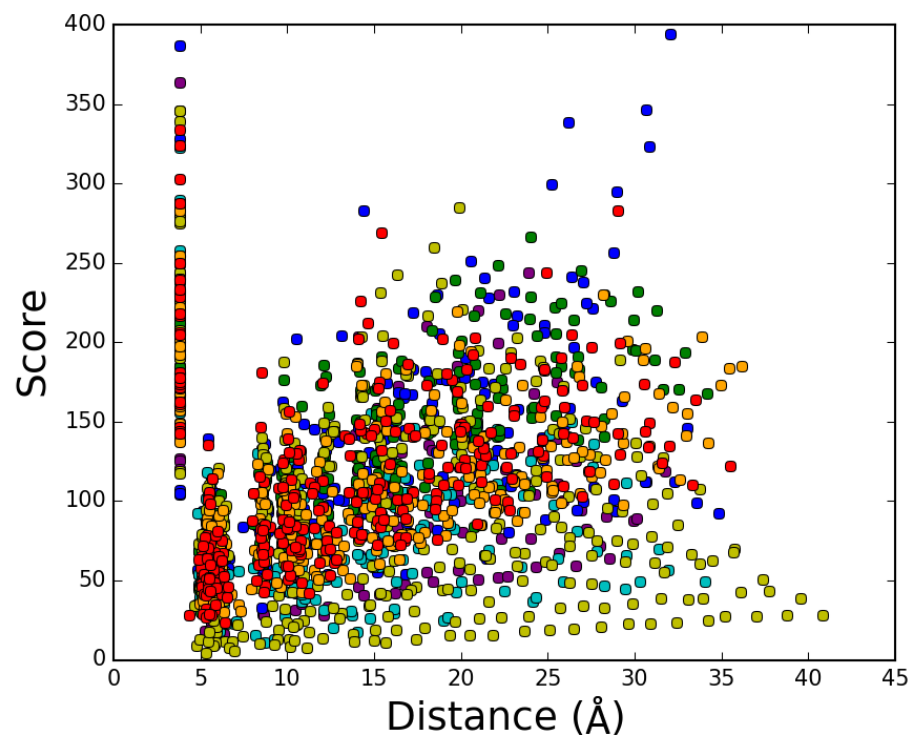

Figure S6. Correlation between score and the average distance for 1,992 intrahelical pairs without a chain obtained from cryo-electron microscopy entry 2AT9 in (A) set 1 and (B) set 2, The pairs are colored as follows; purple, helix A; blue, helix B; cyan, helix C; green, helix D; yellow, helix E; orange, helix F; red, helix G.

Table S1. Distribution of amino acid types obtained from 603 sequences of MRs.

Table S2. Details of structures used as set 1 and 2 (wild-type, dark-state bR).

Table S3. Sequence identity among 13 MRs and 23 GPCRs.

Table S4. Pair wise RMSDs in set 1, set 2, set 3, and GPCRs.

Table S5. Secondary structure types (by DSSP) of 13 MRs. 
1. Zhang F, Vierock J, Yizhar O, et al.: The microbial opsin family of optogenetic tools. Cell. 2011; 147(7): 1446-1457.

PubMed Abstract | Publisher Full Text | Free Full Text

2. Liu X, Ramirez S, Pang PT, et al:: Optogenetic stimulation of a hippocampal engram activates fear memory recall. Nature. 2012; 484(7394): 381-385. PubMed Abstract | Publisher Full Text | Free Full Text

3. Oesterhelt $D$, Stoeckenius $W$ : Rhodopsin-like protein from the purple membrane of Halobacterium halobium. Nat New Biol. 1971; 233(39): 149-152. PubMed Abstract | Publisher Full Text

4. Lanyi JK, Luecke H: Bacteriorhodopsin. Curr Opin Struct Biol. 2001; 11(4): 415-419. PubMed Abstract | Publisher Full Text

5. Sabehi G, Loy A, Jung KH, et al:: New insights into metabolic properties of marine bacteria encoding proteorhodopsins. PLoS Biol. 2005; 3(8): e273. PubMed Abstract | Publisher Full Text | Free Full Text

6. Béjà $O$, Lanyi JK: Nature's toolkit for microbial rhodopsin ion pumps. Proc Natl Acad Sci U S A. 2014; 111(18): 6538-6539.

PubMed Abstract | Publisher Full Text | Free Full Text

7. Ran T, Ozorowski G, Gao Y, et al:: Cross-protomer interaction with the photoactive site in oligomeric proteorhodopsin complexes. Acta Crystallogr D Biol Crystallogr. 2013; 69(Pt 10): 1965-1980.

PubMed Abstract | Publisher Full Text

8. Gushchin I, Chervakov $P$, Kuzmichev $P$, et al.: Structural insights into the proton pumping by unusual proteorhodopsin from nonmarine bacteria. Proc Natl Acad Sci U S A. 2013; 110(31): 12631-12636. PubMed Abstract | Publisher Full Text | Free Full Text

9. Rosenbaum DM, Rasmussen SG, Kobilka BK: The structure and function of G-protein-coupled receptors. Nature. 2009; 459(7245): 356-363. PubMed Abstract | Publisher Full Text | Free Full Text

10. Zhang $X$, Stevens RC, Xu F: The importance of ligands for $G$ protein-coupled receptor stability. Trends Biochem Sci. 2015; 40(2): 79-87. PubMed Abstract | Publisher Full Text

11. Kinoshita M, Okada T: Structural conservation among the rhodopsin-like and other G protein-coupled receptors. Sci Rep. 2015; 5: 9176 PubMed Abstract | Publisher Full Text | Free Full Text

12. Ballesteros JA, Weinstein $\mathrm{H}$ : Integrated methods for the construction of three-dimensional models and computational probing of structure-function relations in G protein-coupled receptors. Methods Neurosci. 1995; 25: 366-428. Publisher Full Text
13. Krishnamani V, Lanyi JK: Molecular dynamics simulation of the unfolding of individual bacteriorhodopsin helices in sodium dodecyl sulfate micelles. Biochemistry. 2012; 51(6): 1061-1069. PubMed Abstract | Publisher Full Text

14. Butt HJ, Fendler K, Bamberg E, et al:: Aspartic acids 96 and 85 play a central role in the function of bacteriorhodopsin as a proton pump. EMBO J.1989; 8(6): 1657-1663. PubMed Abstract | Free Full Text

15. Kataoka M, Kamikubo H, Tokunaga $F$, et al:: Energy coupling in an ion pump. The reprotonation switch of bacteriorhodopsin. J Mol Biol. 1994; 243(4): 621-638. PubMed Abstract | Publisher Full Text

16. Rossman MG, Liljas A: Letter: Recognition of structural domains in globular proteins. J Mol Biol. 1974; 85(1): 177-181.

PubMed Abstract | Publisher Full Text

17. Jones TA, Thirup S: Using known substructures in protein model building and crystallography. EMBO J. 1986; 5(4): 819-822. PubMed Abstract | Free Full Text

18. Schneider TR: Objective comparison of protein structures: error-scaled difference distance matrices. Acta Crystallogr D Biol Crystallogr. 2000; 56(Pt 6): 714-721. PubMed Abstract | Publisher Full Text

19. Thompson JD, Higgins DG, Gibson TJ: CLUSTAL W: improving the sensitivity of progressive multiple sequence alignment through sequence weighting, position-specific gap penalties and weight matrix choice. Nucleic Acids Res. 1994; 22(22): 4673-4680. 1994; 22(22): 4673-4680.
PubMed Abstract | Publisher Full Text | Free Full Text

20. Hall TA: BioEdit: a user-friendly biological sequence alignment editor and analysis program for Windows 95/98/NT. Nucleic Acids Symp Ser. 1999; 41: 95-98. Reference Source

21. McNicholas S, Potterton E, Wilson KS, et al.: Presenting your structures: the CCP4mg molecular-graphics software. Acta Crystallogr D Biol Crystallogr. 2011; 67(Pt 4): 386-394.

PubMed Abstract | Publisher Full Text | Free Full Text

22. Asano M, Ide S, Kamata A, et al.: Dataset 1 in: Sequence and intramolecular distance scoring analyses of microbial rhodopsins. F1000Research. 2016a. Data Source

23. Asano M, Ide S, Kamata A, et al.: Dataset 2 in: Sequence and intramolecular distance scoring analyses of microbial rhodopsins. F1000Research. 2016b. Data Source 


\section{Open Peer Review}

\section{Current Peer Review Status:}

\section{Version 1}

Reviewer Report 14 March 2016

https://doi.org/10.5256/f1000research.8526.r12405

(C) 2016 Sugihara $\mathbf{M}$. This is an open access peer review report distributed under the terms of the Creative Commons Attribution License, which permits unrestricted use, distribution, and reproduction in any medium, provided the original work is properly cited.

\section{Minoru Sugihara}

Bioinformatics, Meiji Pharmaceutical University, Tokyo, Japan

In this manuscript, Asano et al. have applied the own developed method, SDS, to microbial rhodopsins. The basic idea of this scoring is to calculate all Carbon-alpha pairwise distances in each crystal structure and to avoid the ambiguity from the structure-matching.

The manuscript is well presented and the method/results will be of interest to broad readers. I would recommend it for acceptance.

Some minor comments:

Authors propose a new numbering scheme (*.50) from the conservation rate of residues. Adding conservation rates of key residues to the legend of Fig.S1 or Table 1 is useful (one need not to check Table S1). Also in Figure S1 only the residue number " 216 " of Helix 7 (7.50) is shown and other numbers are missing.

The different numbering in Figure $\mathrm{S} 1$ and Figure $\mathrm{S} 2$ is a bit confusing. Adding the numbers of the *.50 residue in the bR sequence $(27,57,82,122,152,182$, and 216$)$ at the bottom of the sequencing alignment (Figure S2) might be helpful.

Competing Interests: No competing interests were disclosed.

\section{I confirm that I have read this submission and believe that I have an appropriate level of expertise to confirm that it is of an acceptable scientific standard.}

Author Response ( F1000Research Advisory Board Member ) 24 Mar 2016

Tetsuji Okada, Gakushuin University, Tokyo, Japan

Thank you for the critical reading and valuable comments on our DSA (not SDS) method. According to your suggestions, we have revised Table 1, Figures S1 and S2. An accidental 
error found in the previous Table S1 is also resolved.

Competing Interests: No competing interests were disclosed.

Reviewer Report 01 March 2016

https://doi.org/10.5256/f1000research.8526.r12678

(C) 2016 Navarro J. This is an open access peer review report distributed under the terms of the Creative Commons Attribution License, which permits unrestricted use, distribution, and reproduction in any medium, provided the original work is properly cited.

\section{Javier V. Navarro}

Department of Neuroscience \& Cell Biology, University of Texas Medical Branch, Galveston, TX, USA

This article describes a novel structural analysis of microbial photoreceptors by scoring intramolecular distances derived from their high resolution crystal structures. This work could potentially provide the structural foundation to explain the diverse photoreceptor phenotypes, including identifying the structural factors governing their colour tuning, which is still an unresolved problem.

Competing Interests: No competing interests were disclosed.

I confirm that I have read this submission and believe that I have an appropriate level of expertise to confirm that it is of an acceptable scientific standard.

Author Response ( F1000Research Advisory Board Member) 02 Mar 2016

Tetsuji Okada, Gakushuin University, Tokyo, Japan

We appreciate the positive referee comment. Further results on the MR subfamilies will be discussed in terms of the color tuning mechanism.

Competing Interests: No competing interests were disclosed. 
The benefits of publishing with F1000Research:

- Your article is published within days, with no editorial bias

- You can publish traditional articles, null/negative results, case reports, data notes and more

- The peer review process is transparent and collaborative

- Your article is indexed in PubMed after passing peer review

- Dedicated customer support at every stage

For pre-submission enquiries, contact research@f1000.com 